\title{
Endovascular Treatment of Subclavian Artery Stenosis: Single-Center Experience
}

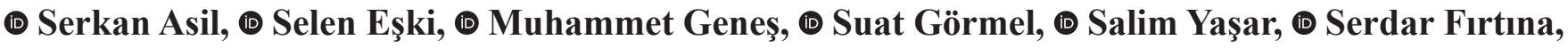 \\ (1) Hatice Tolunay, (1) Uygar Çağdaş Yüksel, (1) Murat Çelik
}

University of Health Sciences Turkey, Gülhane Training and Research Hospital, Clinic of Cardiology, Ankara, Turkey

\begin{abstract}
Objectives: The etiology of subclavian artery stenosis can be very different, but typically the most common form is due to atherosclerotic disease. Interventional treatment is generally indicated for upper limb ischemia, vertebrobasilar symptoms, subclavian steal syndrome, and coronary steal syndrome. Endovascular stenting is preferred over surgery because of its high success rate, less invasive nature, and minimal complication rate. In this study, the characteristics of patients treated endovascularly due to subclavian artery stenosis in our center and their procedural details will be examined.

Materials and Methods: We retrospectively analyzed patients with subclavian artery stenosis treated by endovascular techniques in our center between January 2019 and January 2021.

Results: Twelve (80\%) of 15 patients with stenotic subclavian arteries were successfully treated with endovascular techniques. In 3 patients, the procedure was terminated with failure. The mean age of the patients was 64.66 years and $3(20 \%)$ of the patients were female. All of the patients were receiving antihypertensive treatment with the diagnosis of hypertension, and $4(26.66 \%)$ patients had diabetes mellitus, 13 (86.66\%) patients had hyperlipidemia, and $13(86.66 \%)$ patients had coronary artery disease. In 2 patients, the lesion was diagnosed in the right subclavian artery.

In 7 patients, the procedure was performed with the telescopic method from a transfemoral approach. In 5 patients, the transradial approach was used. In 3 patients, the procedure was performed directly over the transbrachial approach.
\end{abstract}

Address for Correspondence: Serkan Asil, University of Health Sciences Turkey, Gülhane Training and Research Hospital, Clinic of Cardiology, Ankara, Turkey

e-mail: dr_serkanasil@hotmail.com ORCID: orcid.org/0000-0002-6782-4237

Received: 27.08.2021 Accepted: 14.09.2021

Cite this article as: Asil S, Eşki S, Geneş M, Görmel S, Yaşar S, Fırtına S, Tolunay H, Yüksel UÇ, Çelik M. Endovascular Treatment of Subclavian Artery Stenosis: Single-Center Experience. EJCM 2021;9(3):136-142.

DOI: 10.32596/ejcm.galenos.2021-08-045 
Balloon expandable stent implantation was performed in $10(66.66 \%)$ patients at the end of the procedure, and selfexpandable stent implantation was performed in 1 patient.

Conclusion: In conclusion, signs and symptoms can be managed effectively with endovascular techniques in patients with significant subclavian artery disease. In this study, we shared our real-life data on subclavian artery endovascular treatment.

Keywords: Subclavian artery disease, endovascular treatment, patient characteristic

\section{Introduction}

The etiology of subclavian artery stenosis may be multifold; however, it is typically due to atherosclerotic disease and is initially suspected when an inter-arm brachial systolic blood pressure (SBP) difference is present in physical examination ${ }^{(1)}$. However, other causes such as dissection, radiation-induced inflammation fibromuscular disease, and various vasculitides, especially Takayasu arteritis, are not infrequent ${ }^{(2)}$. Generally, the left subclavian artery is about four times more likely to be affected than the right subclavian artery ${ }^{(3)}$. The cases are usually over the age of 50 years. It is seen 1.5-2 times more in men than in women. Subclavian artery disease is usually focal and the lesion is mostly in the first $2 \mathrm{~cm}$ proximal segment of the vessel from the aortic origin.

Stenosis of the subclavian artery alone is usually asymptomatic due to the rich collateral circulation in the head and neck vessels. Intervention is generally indicated for the upper limb ischemia, vertebrobasilar symptoms, subclavian steal syndrome, and coronary steal syndrome in which stenosis proximal to internal mammary-coronary artery bypass may cause ischemic symptoms. The preferred treatment modality for subclavian stenosis, which can be treated surgically and endovascularly, is endovascular treatment with low complication rates and high success rates. The first reported successful endovascular treatment of subclavian stenosis was done by Bachman and Kim in $1980^{(4)}$. Since that time, numerous other reports have been published in the literature with varying degrees of procedural success. We report here our series of 15 patients who underwent intraluminal balloon dilatation and stent implantation of the subclavian stenosis. In this article, it was tried to draw attention to subclavian artery stenosis, which is a rare site of atherosclerosis, it was planned to discuss endovascular treatment methods, feasibility and complications of the procedure were emphasized.

\section{Materials and Methods}

The study was planned as a retrospective registry study. Patients who underwent endovascular treatment for subclavian artery stenosis in our center between January 2019 and January 2021 and whose data could be accessed were included in the study. Patients whose data could not be reached were excluded and no additional exclusion criteria were determined.

The study was approved by the University of Health Sciences Turkey, Gülhane School of Medicine Ethics Committee (date: 20/05/2021, decision no: 2021/231).

\section{Diagnosis and Preprocedural Evaluation}

The difference of more than $15 \mathrm{mmHg}$ between systolic blood pressures measured in both arms, weakness or absence of a pulse in one arm suggests subclavian artery occlusive disease. The use of doppler ultrasonography for scanning in subclavian artery stenosis and occlusion is a cost-effective and non-invasive screening method. Monophasic post-stenotic flow and reversal of systolic flow in the ipsilateral vertebral artery suggest the presence of $>70 \%$ stenosis in the proximal segment of the subclavian $\operatorname{artery}^{(5)}$. In the presence of abnormal or suspicious 
ultrasound findings, advanced anatomical imaging should be considered. Although digital subtraction angiography (DSA) is considered the gold standard imaging method, today computed tomography angiography (CTA) and magnetic resonance angiography (MRA) are used with high specificity and sensitivity rates and a success rate equal to conventional angiography ${ }^{(5)}$.

The diagnosis was confirmed by CTA before the procedure in our patient's cohort with suspected subclavian artery stenosis. At the same time, with CTA, lesion characteristics, degree of calcification, collateral circulation, the relationship between vertebral artery, left internal mammary artery (LIMA) and subclavian artery stenosis can be evaluated. This is very important for determining the technique before the process and choosing the material to be used, increasing the success of the process (Figure 1).

Risk factor control is recommended in all patients with subclavian artery occlusive disease to reduce cardiovascular risk $^{(5)}$. For this purpose, for every patient who did not use antiaggregant and statin therapy, acetylsalicylic acid and high-intensity statin therapy were initiated. Other atherosclerosis risk factors of the patients were controlled. At the same time, symptom-based investigations were conducted for coronary, carotid and lower extremity arterial diseases.

\section{Procedural Details}

For all patients, the procedures were performed in the catheter laboratory under local anesthesia. In most cases, the anterograde transfemoral approach is used as the first choice. Once arterial access is established, 5000-8000 IU heparin is administered intravenously.

In our cohort, the procedure was performed with the telescopic method from a transfemoral approach in seven patients. For this purpose, we used 7 or $8 \mathrm{Fr}$ destination sheath and multi-purpose catheter or right Judkins catheter. The schematic view of the transfemoral approach is described in Figure 2. In five patients, the transradial approach was used, first lesion canalization was performed with 0.014 inches $300 \mathrm{~cm}$ coronary wire. After the wire was snared in the descending aorta, the procedure was performed with the help of a destination sheath from the transfemoral approach. In three patients, the procedure was performed directly over the transbrachial approach.

In subclavian arteries intervention, the guidewire selection is performed based on the lesion feature and the sheath/guide catheter planned to be used. Conventionally, a $0.028-0.032$ inches (at least $260 \mathrm{~cm}$ long) guidewire is used to pass moderate stenosis and provide adequate support throughout the procedure. If necessary, for higher grade strictures and complete occlusions, flat-tipped hydrophilic wires and 0.014-inch or 0.018-inch guidewires on the
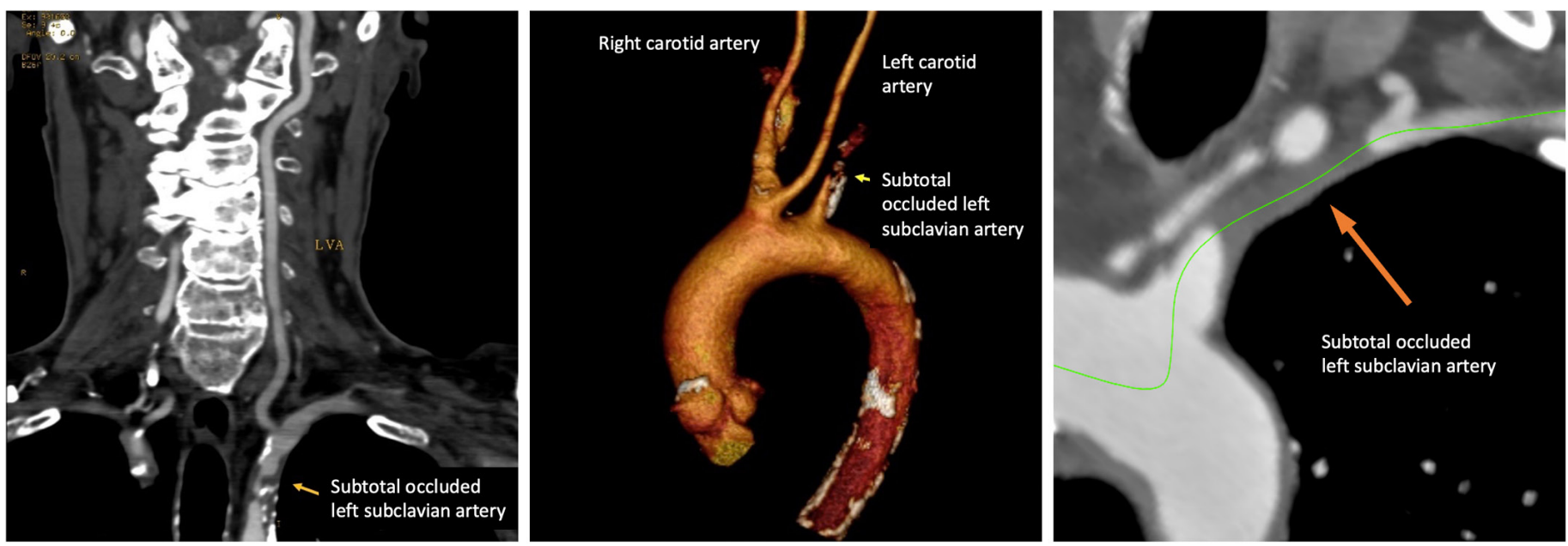

Figure 1. Computed tomographic angiography evaluation before the procedure 


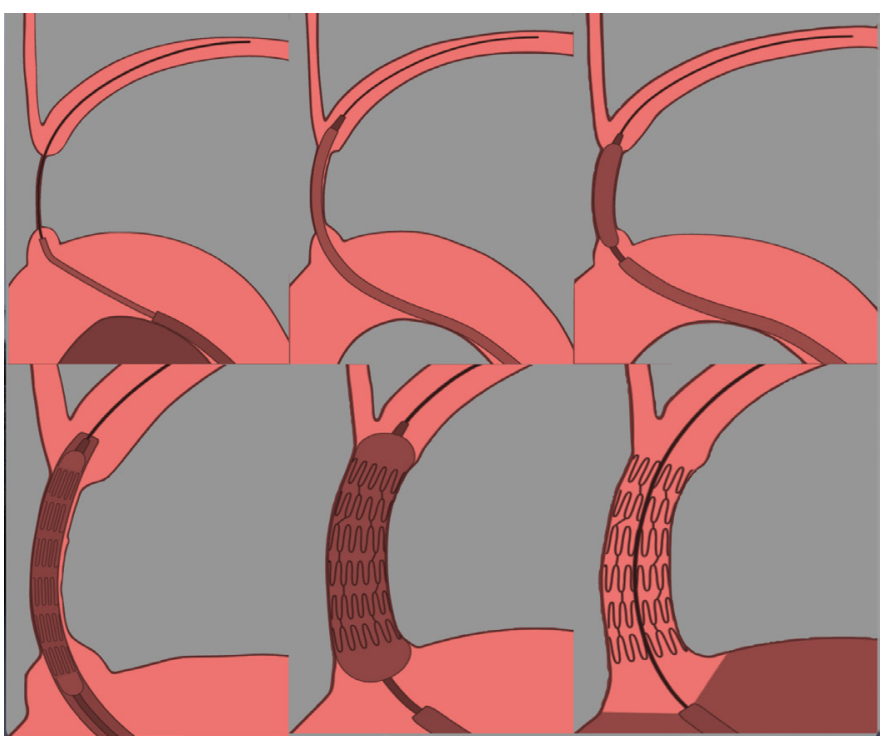

Figure 2. Step by step schematic view of the transfemoral approach

microcatheter support can be used. Stenting, especially balloon-expandable stents, is generally preferred over alone balloon angioplasty in the treatment of subclavian artery stenosis.

\section{Results}

The mean age of the patients was 64.66 years and 3 $(20 \%)$ of the patients were female. The risk factors in this population included hyperlipidemia (13 patients $86.6 \%$ ), diabetes mellitus (4 patients, $26.6 \%$ ), hypertension (15 patients, $100 \%$ ), and smoking ( 8 patients $53.3 \%$ ). Coronary artery diseases were present in 13 patients $(86.8 \%)$; five patients had undergone a coronary artery bypass, of which all had a bypass done using the LIMA (Table 1).

Symptomatology included subclavian steal syndrome and vertebrobasilar insufficiency in nine patients $(60 \%)$, coronary steal syndrome and protection of a LIMA coronary bypass in five patients (33.3\%). Subclavian aneurysm, which is a rare clinic, was our indication in one of our patients (Table 1). Twelve (80\%) of 15 patients with subclavian artery stenosis were successfully treated with endovascular methods. In three patients, the procedure was terminated unsuccessfully. The procedure was terminated early because a stroke developed in the subclavian aneurysm patient during the procedure. She recovered without sequelae with anticoagulant therapy in the follow-up. In one patient, the stenosis could not be passed because of a heavily calcified lesion. In the third patient, the procedure was terminated due to heavy calcification and subintimal dissection extending to the brachial artery and thrombus development. These patients, in whom the procedures failed, were started to be followed up under medical treatment because they did not want to undergo surgery, and if they became symptomatic despite medical treatment, it was decided to reschedule endovascular treatment. While the endovascular intervention was performed to a total occluded artery in 8 patients, other patients lesions were not total occluded. Two patients had right subclavian and 13 patients had left subclavian artery disease (Table 1).

Balloon expandable stent implantation was performed in $10(66.66 \%)$ patients at the end of the procedure, and self-expandable stent implantation was performed in one patient. One patient with subclavian stent restenosis was treated only with percutaneous transluminal angioplasty (Table 1).

\section{Discussion}

In this study, we shared our real-life experience on subclavian artery endovascular treatment. The basic demographic characteristics of the patients, as well as the technical details of my procedure, were explained. Endovascular therapy has become the primary treatment approach for symptomatic subclavian artery disease. Although surgical revascularization is the preferred treatment option in guidelines and textbooks due to acceptable long-term results, percutaneous endovascular approaches have come to the fore in recent years due to high success rates, long-term patency and low complication rates, shorter hospital-stay and significantly lower cumulative costs compared to surgical treatment ${ }^{(6)}$. Conventional surgical revascularization can also be performed intrathoracically concurrently with cardiac 


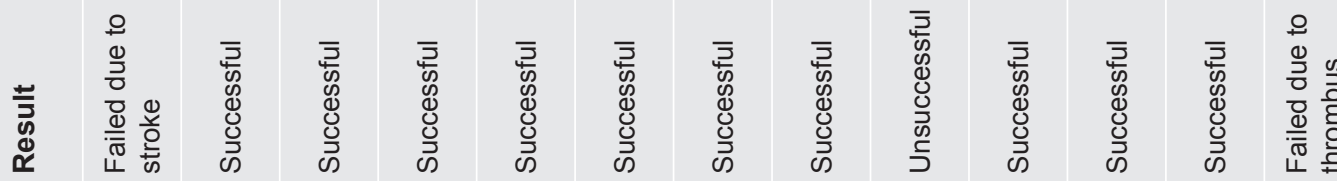

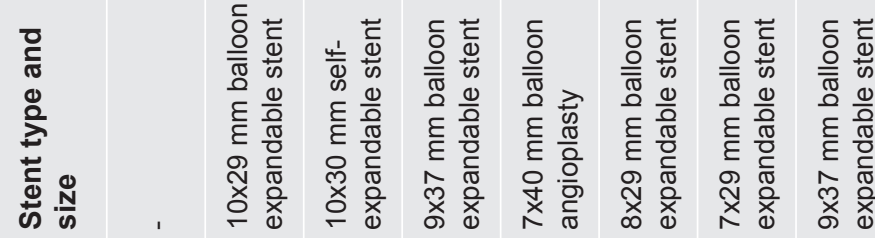

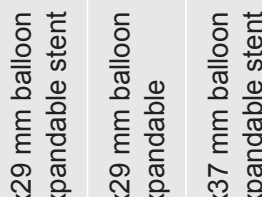

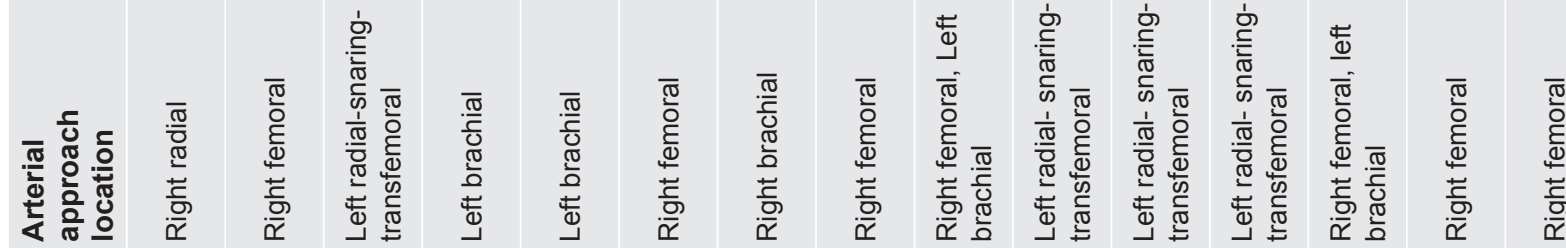

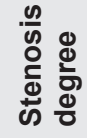

@
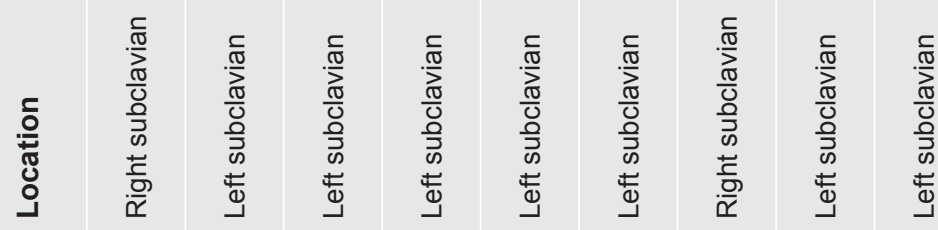

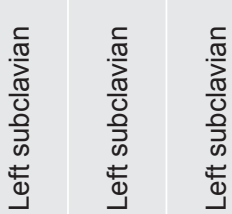

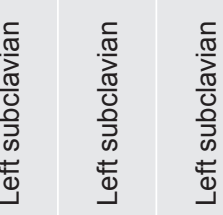

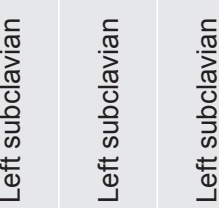

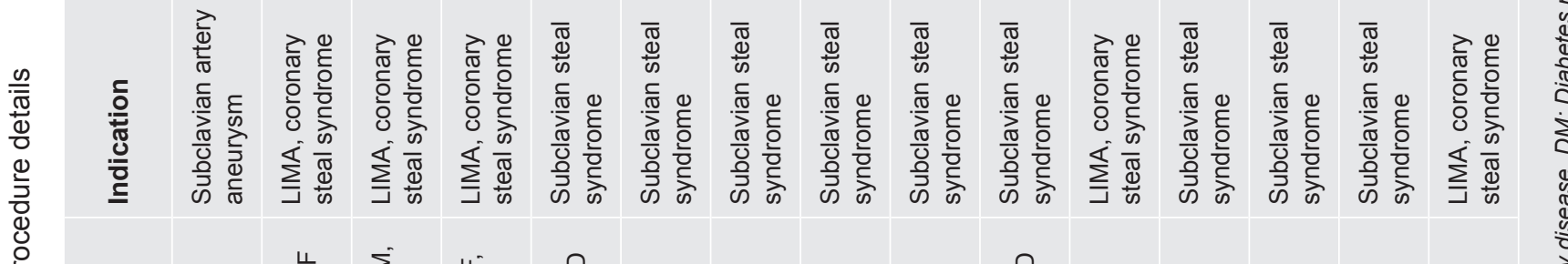

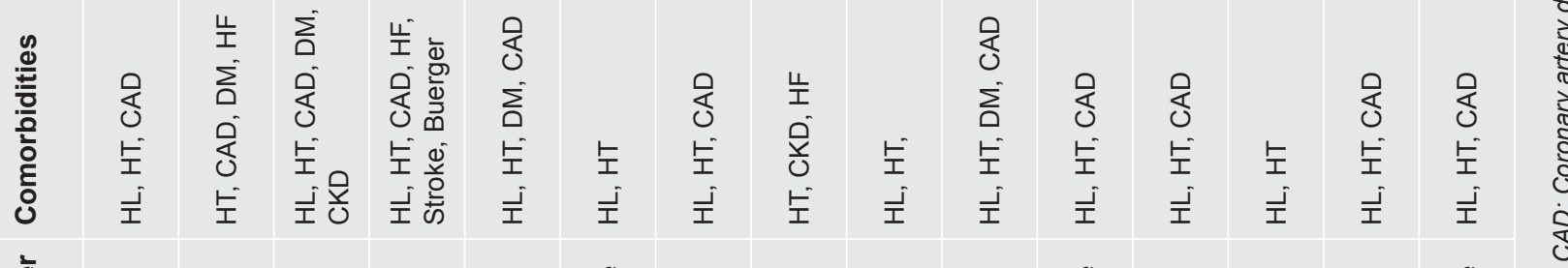

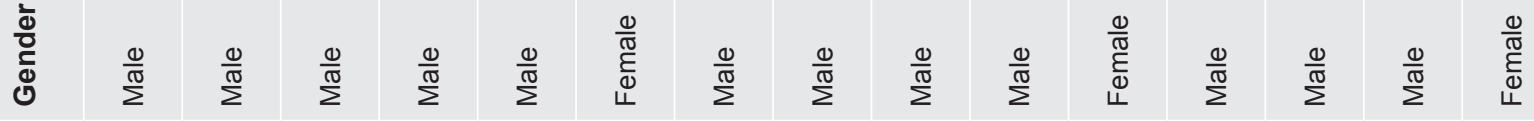


surgery or extra-thoracically as carotid-subclavian, carotid-axillary, subclavian-subclavian and axilla-axillary bypass $^{(7)}$. Among the surgical revascularization techniques used, carotid-subclavian bypass seems to be more advantageous due to a shorter anastomosis and relatively good patency rates ${ }^{(7)}$.

Depending on the location and severity of the lesion, anterograde (femoral), retrograde (brachial/radial) or combined methods may be preferred in endovascular treatment. The femoral artery approach should be chosen in subtotal lesions with sufficient stump in the proximal subclavian artery. Due to the high fibrocalcific nature of lesion and rich collaterals circulation, it is difficult to pass chronic complete occlusion anterogradely. Therefore, retrograde brachial or radial artery approach may be preferred in total occlusions without an osteal stump, in long segment lesions, in the presence of severe tortuous aorta, in cases where the exit angle of the subclavian artery from the aortic arch is abnormal.

There is no definitive evidence as to whether stenting is more effective than balloon angioplasty alone in subclavian artery occlusive diseases. However, in a systematic review (544 patients) comparing both options, stenting was found to be superior with higher patency rates compared to angioplasty alone ${ }^{(8)}$. Balloon-expandable stents are preferred rather than self-expandable stents to implant the stent with appropriate sensitivity in lesions in the osteal and proximal regions because there is a possibility of stent dislocation and migration in self-expanding and undersized stents. Moreover, in severe calcified osteal lesions, in addition to easier insertion, balloon-expandable stents provide greater radial force than self-expandable stents. In cases where the lesion is after the vertebral artery, balloon angioplasty should be preferred first, as there is a risk of bending and compression of the stent at the thoracic outlet, especially between the first rib and clavicle. When the lesion does not respond to balloon angioplasty, selfexpanding nitinol stents are more appropriate to avoid the possibility of compression by extravascular structures.

While the technical success rate of endovascular treatment in subclavian artery occlusive disease is quite high (97-100\%) for stenosis, the success rate for incomplete occlusions decreases because of the length of the occluded segment and the intensity of calcification $(46-76 \%)^{(6)}$. De Vries et al. ${ }^{(9)}$, reported in their case series of 110 patients over 10 years that stenting was implanted in $58 \%$ of patients with a $93 \%$ technical and clinical success and $1 \%$ complication rate in the endovascular treatment of symptomatic subclavian artery stenosis. In our case series, twelve $(80 \%)$ of 15 patients with subclavian artery stenosis were successfully treated with endovascular methods. In three patients, the procedure was terminated unsuccessfully. In a patient who had a subclavian aneurysm, the procedure was terminated early because stroke developed during the procedure, and the other two failed due to total calcific occlusion. In the literature, although long-term follow-up data are insufficient, long-term patency (3-5 years) rate in stenosis is $84 \%$. This rate has been reported as $64 \%$ for complete occlusions ${ }^{(10)}$. In-stent restenosis is a major problem with endovascular procedures. Because the subclavian artery is an elastic and big vessel, the recurrence rate is relatively low and ranges from $5 \%$ to $7 \%{ }^{(11)}$.

Complication rates ranging from $3 \%$ to $11 \%$ have been reported with endovascular treatment of subclavian artery stenosis ${ }^{(6)}$. Reported complications include subclavian artery thrombosis, axillary artery thrombosis, stent migration, stent dislocation, flow-limiting arterial dissection, distal embolization, arterial extravasation, arterial access complications, hematoma requiring transfusion, restenosis, and neurological complications (transient ischemic attack, stroke, hemiplegia, diplopia etc.). In our own case series, complications were observed in two patients $(13.33 \%)$, the patient with subclavian aneurysm had a stroke complication, and another patient had complications of brachial artery dissection and thrombosis.

\section{Study Limitations}

Our study has many limitations. The most important of them is the small number of patients. Another important limitation is that the study was planned retrospectively. 
Although the study has very important limitations; we think that it is important that it is a real-life experience and that the etiology, diagnosis, preprocedural evaluation of subclavian artery stenosis and the technical details of the procedure are explained.

\section{Conclusion}

Depending on the technological developments in endovascular interventions and the increase in operator experience, endovascular treatment in subclavian artery occlusion has become the primary treatment due to low complication and high success rate. In this study, the experience of our center in this field was transferred based on patient and procedural characteristics.

\section{Ethics}

Ethics Committee Approval: This study was approved by the University of Health Sciences Turkey, Gülhane School of Medicine Ethics Committee (date: 20/05/2021, decision no: 2021/231).

Informed Consent: Retrospective study.

Peer-review: Externally peer-reviewed.

\section{Authorship Contributions}

Concept: S.A., S.E., Design: S.A., M.Ç., S.G., Data Collection or Processing: S.E., M.G., S.G., S.F., S.Y., Analysis or Interpretation: S.A., M.Ç., U.C.Y., Literature Search: S.A., S.Y., S.F., H.T., U.Ç.Y., Writing: S.A., M.Ç.

Conflict of Interest: No conflict of interest was declared by the authors.

Financial Disclosure: The authors declared that this study received no financial support

\section{References}

1. Shadman R, Criqui MH, Bundens WP, et al. Subclavian artery stenosis: prevalence, risk factors, and association with cardiovascular diseases. J Am Coll Cardiol 2004:44:618-23.

2. Ochoa VM, Yeghiazarians Y. Subclavian artery stenosis: a review for the vascular medicine practitioner. Vasc Med 2011;16:29-34

3. Rodriguez-Lopez JA, Werner A, Martinez R, Torruella LJ, Ray LI, Diethrich EB. Stenting for atherosclerotic occlusive disease of the subclavian artery. Ann Vasc Surg 1999;13:254-60.

4. Bachman DM, Kim RM. Transluminal dilatation for subclavian steal syndrome. AJR Am J Roentgenol 1980;135:995-6.

5. Aboyans V, Ricco JB, Bartelink MEL, et al. 2017 ESC Guidelines on the Diagnosis and Treatment of Peripheral Arterial Diseases, in collaboration with the European Society for Vascular Surgery (ESVS): Document covering atherosclerotic disease of extracranial carotid and vertebral, mesenteric, renal, upper and lower extremity arteriesEndorsed by: the European Stroke Organization (ESO)The Task Force for the Diagnosis and Treatment of Peripheral Arterial Diseases of the European Society of Cardiology (ESC) and of the European Society for Vascular Surgery (ESVS). Eur Heart J 2018;39:763-816.

6. Patel SN, White CJ, Collins TJ, et al. Catheter-based treatment of the subclavian and innominate arteries. Catheter Cardiovasc Interv 2008;71:963-8.

7. Aydın C, Ay Y, Kara İ, et al. Subklavyen Arter Tıkanıklığında Cerrahi Revaskülarizasyon Uygulamalarımızın Erken-Orta Dönem Sonuçları. Koşuyolu Kalp Dergisi 2013;16:19-24.

8. Chatterjee S, Nerella N, Chakravarty S, Shani J. Angioplasty alone versus angioplasty and stenting for subclavian artery stenosis--a systematic review and meta-analysis. Am J Ther 2013;20:520-3.

9. De Vries JP, Jager LC, Van den Berg JC, et al. Durability of percutaneous transluminal angioplasty for obstructive lesions of proximal subclavian artery: long-term results. J Vasc Surg 2005;41:19-23.

10. AbuRahma AF, Bates MC, Stone PA, et al. Angioplasty and stenting versus carotid-subclavian bypass for the treatment of isolated subclavian artery disease. J Endovasc Ther 2007;14:698-704.

11. Heuser RR, Henry M. Textbook of peripheral vascular interventions: Taylor \& Francis US; 2008 